\title{
LA INTEGRACIÓN REGIONAL LATINOAMERICANA A PARTIR DE LAS OPORTUNIDADES PARA EL PROGRESO ${ }^{1}$ \\ THE LATIN AMERICAN REGIONAL INTEGRATION BASED ON ITS OPPORTUNITIES FOR PROGRESS
}

\author{
Jhoner Perdomo \\ Universidad Central de Venezuela, Venezuela \\ jhonerperdomo@yahoo.com \\ Luis Daniel Álvarez \\ Universidad Central de Venezuela, Venezuela \\ luis.daniel.alvarez.v@gmail.com \\ Sary Levy-Carciente \\ Universidad Central de Venezuela, Venezuela \\ saryle@yahoo.com \\ Mauricio Phélan \\ Universidad Central de Venezuela, Venezuela \\ mauphelan@gmail.com
}

Cómo citar / Citation

Perdomo, Jhoner; Álvarez, Luis Daniel; Levy-Carciente, Sary y Phélan, Mauricio (2016). "La integración regional latinoamericana a partir de las oportunidades para el progreso". OBETS. Revista de Ciencias Sociales, 11(2): 513-545. doi:10.14198/OBETS2016.11.2.06

\section{Resumen}

El objetivo de un Estado debería ser la ampliación de oportunidades y capacidades para que la ciudadanía promueva resultados que permitan el desarrollo humano. En este sentido, los países se integran en bloques para fortalecer sus políticas, dar respuestas a las demandas sociales y mejorar la calidad de

\footnotetext{
${ }^{1}$ Artículo elaborado en el marco del Proyecto CDCH-UCV PG-05-8650-2013/2.
} 
vida de sus habitantes. Por ello, la conformación de alianzas presume que quienes las conforman poseen elementos y objetivos en común. Se realiza un ejercicio a países de la región latinoamericana, mediante un análisis discriminante utilizando indicadores multidimensionales, cuyo objetivo es determinar cuáles son las condiciones que permiten diferenciar los respectivos convenios en la región, para nuestro análisis: MERCOSUR, ALBA y Alianza del Pacifico.

Palabras Claves: Integración Regional; Análisis Discriminante; Indicadores; MERCOSUR; ALBA y Alianza del Pacifico.

\begin{abstract}
The goal of a state should be expanding opportunities and capabilities citizenship to promote results that enable human development. In this regard, countries integrate into blocks to strengthen their policies, to respond to social demands and improve the quality of life of its inhabitants. Therefore the formation of alliances presumes that those who make them possess elements and objectives in common. An exercise is performed to countries in the Latin American region, using the discriminant analysis using multidimensional indicators, which aims to determine what are the conditions that allow differentiating blocks in the region, for our analysis: MERCOSUR, ALBA and Pacific Alliance.
\end{abstract}

Keywords: Regional integration; discriminant analysis; indicators; MERCOSUR; ALBA and Pacific Alliance.

\title{
Extended abstract
}

In a global world, Latin American countries cannot act as independent agents from the rest of the world. From the multidimensional approach of development, conditions that encourage and expand opportunities and capabilities so that citizenship could promote results that enable human development, have to be created. To favor the achievement of these goals, regional integration agreements are valuable mechanisms. The alliances formed in Latin America look for unifying efforts with common purposes and, at first, their members seemto have very homogeneous features. While the conformation of alliances express different interests -that can be of economic or political orderthe intention of the study is to analyze if in the groupings and their countries the populations' capabilities and opportunities are similar among them.

With the aim of studying the performance of several integration agreements worldwide, a very useful method is the Discriminant Analysis. Using selected indicators, itseeks to define the features that significantly distinguisha group, it allows consideringmembers of a group and to analyze its homogeneous or heterogeneous behavior in them, as well as validate significant differences with other groups or agreements. The Step by Steptechnique uses only those indicators with more influence, seeking to represent a discriminant function with the least amount of possible variables and which is equally significant to distinguish groups. For this reason the selected variables are those that provide centroids further away among groups and more concentrated within groups. In this sense, the technique reflects the result of the innerbehavior of each 
grouping, agreement or alliance, reflecting if its members have more or less commonalities, fostering its integration or rather the possibility to split up. In addition, the statistical test of contrast and probability of membershipshows the statistical probability of belonging to the original group or to some other group, and thus validate the consistency of the existing groupings.

For 19 countries of Latin America that make up the selected case studies: Southern Common Market (MERCOSUR), Bolivarian Alliance for the Peoples of our America (ALBA)and Pacific Alliance (AP), the data selected included indexes associated with welfare, civil liberties, political, economic, and environmental aspects to depict a multidimensional profile of each of the countries and of the respective blocks or associations, with an alternative perspective of development.

There were identified 60 indices that measure, in one way or another, environmental, socio-economic, political, technological and institutional aspects. From the list of indices,in a first moment were selected those with complete information for all Latin American countries. In a second step, it was made a comparative analysis of their dimensions to reduce the multiplied presence of the same dimension and to avoid some type of covariance between the selected indicators. Finally 10 indices were considered: Institutional Quality, Property Rights, Cost of Living, Democracy, Complexity, Misery, Social Progress, Global Innovation, Globalization and Fragile State; all multidimensional and largely associated with the dimensions established for the UN's sustainable development goals by the year 2030 .

Two statistical tests were performed among groups, the first among ALBA, AP and the rest of the countries of Latin America; the second among MERCOSUR, AP and the rest of the countries of Latin America. In the case of the first and second test, of the 10 variables under analysis, 6 and 7 had discriminatory power, respectively. Of them Global Innovation Indexfor the first test -in more favorable terms to Pacific Alliance- and Global Innovation Index and Misery Index for the second test, are enough in both cases to differentiate the groups under analysis and to develop a discriminant model. The fact that not all variables had discriminatory power is indicative that groups are not homogeneousin certain dimensions. By the contrary those with discriminatory power, indicate that those groups should be analyzed starting from those variables, since being more homogeneous in that indicator they allow a better representation of their country members.

The observed results with thestatistical test of contrast and probability of membershipfor the groupings for the first test is 100\% of the ALBA countries are integrated, while in AP is $83.3 \%$ of countries, and the countries of the rest of Latin America could be divided by which of these two agreements of integration have statistically significant characteristics with more association. Same thing happens with groupings of the second statistical test, whose results show an AP 100\% integrated and MERCOSUR 40\%.

These results indicate that MERCOSUR has heterogeneous features among its members, which in the long run could generate difficulties for the implementation of common policies. Instead, AP and ALBA while representin 
ghighly different alternatives, they are more stable groups, given the homogeneity of their countries members. The difference between them is the kind ofprojectthat guides them more or less towards a progress stage. While the analyzed groupings do not show harmony in every dimension and that still among countries that make up a particular groupthere are differences in some indicators, the results guide to the areas where each group must emphasize in their policies

Although the Latin American regionis very heterogeneous, some indexes threw certain inner homogeneity, especially the Innovation GlobalIndex that appears as a strong screener in those three agreements analyzed. This multidimensional index combines the quality of the institutional and political environment, regulatory and business stability, freedoms of expression and action, investment in humantalent, research and development, innovationlinks, know ledge absorption, impact, creation and dissemination. This way is a measurement that reflects the opportunities for development of a country. Innovation also attractscapital investment, generating employment and increasing economicgrowth, which reduces the Misery.Also within the components of the Innovation Global Index are the regulatoryenvironment, law enforcement, third leveleducation and ecological sustainability. This combination is not far behind the Agenda 2030 goals established by the UN that statesthe importance to foster innovation, promote a sustainable industrialization in balance with nature and besides to have a flexible infrastructure, transparency, rule of law and law enforcement.

By this, innovation is not only a keyelement for the integration of countries, but a fundamentalfactor for its progress. The difference in innovation levels between AP and ALBA are very important, not so much for the case of AP and MERCOSUR, however by 2014 all AP members are in better position to do business compared to countries belonging to MERCOSUR, which are, in very unfavorable positions according to the World Bank Report Doing Business 2014. This means that much emphasis must be done not only in innovation per se, but also in all the component of the index to promote common policies in the integration agreement aligned with Latin American progress, in a way that the process of innovation will weave ties of cooperation among several entities to guarantee a greater success. Groups must make great efforts to consolidate development strategies, with transfer of technology and training human talent altogether.

Thus, scientific innovation appears as the variable that synthesizes the ability of countries to leverage their development. It is worth saying that this innovation is based on a free society in which the exchange of ideas is possible without limitations, and where social arrangements encourage and spread it. Innovation shows the largest differences in the region: those groups that consolidate best strategies aligned to innovation, strong institutions and freedoms will have greater opportunity for their countries to obtain more favorable results for their citizens. By the contrary, groups with minor advances in that field will result in poor opportunities for the citizenship. The integration of Latin America should be comprehensive and uncut, and to emerge as 
a region one of the stages that must be overcome is the technological dependence and taking innovation as the cornerstone for their progress.

\section{REFERENTES TEÓRICOS E HISTÓRICOS SOBRE INTEGRACIÓN REGIONAL}

Desde fines del siglo XX se manifiesta a nivel mundial la convivencia de tendencias globalizadoras con las de integración regional, y en las que la intervención de diversos actores, (Estado-Nación, organizaciones no gubernamentales, empresas privadas y públicas y consumidores) conjuga intereses que influyen de manera determinante en los comportamientos que terminan definiendo la estructura organizacional de nuestro mundo y su desempeño. Los noventa mostrando un incremento de los procesos de integración, concluyendo los estudiosos del enfoque regional que ha surgido una nueva estructura en la que aparecen novedosas formas de institucionalización, crecimiento geográfico, redimensión de los enfoques, haciendo que se deje de lado la figura estrictamente económica, pasando a incluirse los derechos de propiedad intelectual y el tema ambiental; todo bajo un marco de fomento y protección a los derechos humanos. Los nuevos mecanismos de integración incluso han dejado atrás la noción de diplomacia cerrada y han pasado a establecer modelos de transparencia en los que la sociedad civil ejerce una labor de contraloría (De Lombaerde, Kingah y Rettberg, 2014).

Entre las corrientes que explican los mecanismos de integración está el neofuncionalismo que centra sus argumentos en la ganancia o pérdida que se presenta al asociarse a otros Estados, presentándose entonces la necesidad de politizar las etapas del proceso, buscando que se analice a profundidad el acercamiento entre los actores. Aunque el deseo es disminuir la influencia del nacionalismo, en las primeras etapas resulta inevitable una marcada presencia del Estado. Frente a esta corriente está la concepción intergubernamentalista que plantea que la existencia de instituciones se genera por los beneficios que los Estados puedan tener de su desempeño. Hay un control del Estado de todas las etapas del proceso de integración, dependiendo entonces el resultado del cálculo que pueda hacer cada uno (Álvarez y Victoria, 2011).

Sin embargo, finalmente no importaría si se presenta un modelo de integración avalado por lo político o lo económico, pues se terminan constituyendo mecanismos que van a tener impacto en lo social, lo financiero, lo político e incluso lo educativo. Si el objetivo es una alianza económica o la estructuración de un mecanismo político, las variables se transformarán para generar una instancia que termine desarrollando objetivos más amplios a los trazados inicialmente. 
Cabe recordar que los fenómenos de integración no son recientes: los primeros asomos de una integración fuerte se establecen hacia 1828 con la unión aduanera entre Prusia y Hesse-Darmstadt. Posteriormente se concreta la unión aduanera de Bavaria-Württemberg, la Unión Comercial de Alemania Central (German Zollverein), la unión impositiva de Alemania del Norte, la Unión monetaria Alemania y finalmente el Reich Alemán. El auge integracionista alcanzará a lo que hoy día es Suiza, cuando en 1848 se crea el mercado integrado y la unión política Suiza. De igual manera ocurre en Italia con el movimiento del Resurgimiento (Risorgimento). Por su parte, Count Paul Leusse en Francia, insiste en el establecimiento de una unión aduanera agrícola entre Francia y Alemania con un comité de aranceles a establecerse en Frankfurt. En Austria el político y economista Alexander Peez planeó una Unión Europea Central (MiddleEuropeanZollverein) que incluía a Francia. Posteriormente, en 1952 se crea la Comisión Europea del Carbón y el Acero y en 1957 se suscribe el Tratado de Roma naciendo la Comunidad Europea (Levy-Carciente y García, 2007).

Al referirnos a América, Andrade (2011) ubica los primeros intentos de integración regional en tratados de inspiración en la CEPAL, con miras a incentivar los vínculos bajo la premisa de favorecer el crecimiento y la industrialización, como proposiciones iniciales para alcanzar un desarrollo total. Estos procesos que se generaron y lograron plasmar una visión que perduró hasta los años ochenta y noventa, se tradujeron en la creación de zonas de libre comercio subregionales, como ALALC y ALADI; MCCA y SICA²; Acuerdo de Cartagena y la Comunidad del Caribe (CARICOM). Incluso la creación en 1975 del Sistema Económico Latino Americano (SELA) es una muestra más del enfoque y de la propuesta de alcanzar un esquema de formulación que no contemplase a los Estados Unidos y Canadá.

Las razones que sustentan la tesis de enfocar la integración desde una visión sustancialmente económica responden a la necesidad de comprender la delicada situación financiera que atravesaba la región. Para el caso de Venezuela, Romero (2005:115) nos comenta:

"Es en este marco sistémico que se desarrollan las políticas exteriores de Herrera y Lusinchi y dentro de ellas las relativas a la defensa y promoción de la democracia venezolana y hemisférica.

Con mayor o menor eficacia y eficiencia, fueron políticas diseñadas e instrumentadas para un tiempo de crisis. Sin embargo, aunque constreñido económicamente y bajo climas políticos domésticos y regionales de tensión y de nuevas visiones

\footnotetext{
${ }^{2}$ Siglas de Asociación Latinoamericana de Libre Comercio, Asociación Latinoamericana de Integración, Mercado Común Centro Americano, Sistema de Integración Centroamericano, respectivamente.
} 
este - oeste, las agendas de defensa y promoción de la democracia de ambos gobiernos continuaron -como señalamos antes- enfatizando los postulados de pluralismo ideológico y la cooperación bilateral y multilateral regional".

La politización del Pacto Andino hacia 1979 permitió darle mayor dinamismo a su estructura al dotarlo de un Parlamento con funciones establecidas, tribunales y de un Consejo Presidencial que pudiese ejecutar algunas acciones y no se limitara a ser un ente protocolar. A esta iniciativa transformada y modificada siguió la creación del Grupo Contadora. Como se evidencia, lo que llevó a los países a unirse no fue enfrentar mancomunadamente el problema económico, sino que la prioridad pasaba por atender la compleja coyuntura de la violencia que agitaba a gran parte de América Central. En palabras del ex-Canciller de Venezuela, Simón Alberto Consalvi:

"El Mecanismo Permanente de Consulta y Concertación Política es la respuesta a los diversos desafíos que tiene planteados América Latina. Sin concertación no hay manera de resolver los problemas que nos son comunes y que requieren, por tanto, de una posición conjunta. No es posible que mientras los países debilitados a las democracias individuales, para usar la expresión del Dr. Kissinger, coordinen sus acciones y definan sus politicas, los países de América Latina sean un archipiélago de indefiniciones". (Consalvi, 1988: 30).

De igual manera, al revisar las palabras de los Presidentes de Latinoamérica asistentes a la Cumbre de Acapulco, México, en noviembre de 1987, queda claro que la integración se contempla como un mecanismo para unificar la voz de la región. De esta forma, Argentina, Brasil, Paraguay y Uruguay dieron forma al MERCOSUR, mientras que Venezuela, Colombia, Perú, Bolivia y Ecuador procuraron un paso más y cambiaron al Pacto Andino por la Comunidad Andina de Naciones (que mantenía la misma estructura del ente fundado en 1969) que asomaba que su aspiración natural era ingresar al Mercado Común del Sur cuando estuviesen dadas las condiciones. Chile por su parte quedó de forma independiente, México optó por acercamientos económicos con Estados Unidos y Canadá, mientras que el Caribe buscó constituir su mercado común y Centro América, junto a República Dominicana, un mecanismo novedoso en el que resalta en desarrollo y estabilidad Costa Rica.

Desde inicios del siglo XXI han proliferado nuevas formas de asociación de países en la región latinoamericana, algunas que responden a criterios ideológicos, mientras otras se asientan en debates y estrategias económico-financieras. Destacan entre ellas los espacios de: ALBA, UNASUR, CELAC ${ }^{3}$, Alianza Pacífico y Petrocaribe.

${ }^{3}$ Siglas de Alianza Bolivariana para los Pueblos de América, Unión de Naciones Suramericanas y Comunidad de Estados Latinoamericanos y Caribeños, respectivamente. 


\section{ESQUEMAS DE INTEGRACIÓN LATINOAMERICANA BAJO ESTUDIO}

En el presente trabajo se revisarán desempeños de los países integrantes de los acuerdos de integración del MERCOSUR, la Alianza del Pacífico y la ALBA, por lo que a continuación se realizará una breve descripción de dichos esquemas.

\subsection{Mercado Común del Sur ${ }^{4}$}

El Mercado Común del Sur, MERCOSUR, es una iniciativa que tuvo sus orígenes en una propuesta bilateral suscrita en Foz de Iguazú, Brasil, el 30 de noviembre de 1985, pero que adquirió la forma que hoy se conoce el 26 de marzo de 1991 con la firma del Tratado de Asunción. Su entrada en funcionamiento a plenitud se concretó el 15 de diciembre de 1995.

"El MERCOSUR es un proceso abierto y dinámico. Desde su creación tuvo como objetivo principal procurar un espacio común que generara oportunidades comerciales y de inversiones a través de la integración competitiva de las economías nacionales al mercado internacional. Como resultado ha establecido múltiples acuerdos con países o grupos de países, otorgándoles, en algunos casos, carácter de Estados Asociados -es la situación de los países sudamericanos-. Estos participan en actividades y reuniones del bloque y cuentan con preferencias comerciales con los Estados Partes. El MERCOSUR también ha firmado acuerdos de tipo comercial, político o de cooperación con una diversa cantidad de naciones y organismos en los cinco continentes".

Los miembros plenos son Argentina, Brasil, Paraguay Uruguay y Venezuela, éste último Estado se adhirió a la iniciativa en el 200955. Bolivia está tramitando su proceso de adhesión, lo cual llevaría al MERCOSUR a contar con más de 290 millones de habitantes, en una extensión territorial de cerca de 15 millones de $\mathrm{km}^{2}$.

En 1998 el MERCOSUR buscó institucionalizarse declarando todo el territorio "Zona de paz y libre de armas de destrucción en masa" y estableciendo el Compromiso Democrático o Protocolo de Ushuaia, que contempla cláusulas de protección a la democracia, la libertad de empresa y la libertad de expresión. En 2005 se estableció un Fondo para la Convergencia Estructural del MERCOSUR (FOCEM) que a través de un aporte anual de cien millones de dólares busca reducir las asimetrías entre los socios. También es de este año

\footnotetext{
${ }^{4}$ Véase www.MERCOSUR.int/

${ }^{5}$ Después de un controversial procedimiento que se aprovechó de la suspensión temporal de Paraguay que atravesaba una crisis interna y cuyo parlamento vetaba el ingreso de Venezuela.
} 
la creación del Parlamento del MERCOSUR, que debe agotar algunas etapas. La idea del MERCOSUR es que todos los parlamentarios sean electos en comicios directos (no designados en segundo grado) y que eventualmente se elijan el mismo día, en una jornada denominada Día del MERCOSUR Ciudadano.

El MERCOSUR es la quinta mayor economía del mundo y posee todas sus costas en el Océano Atlántico y aunque ha desarrollado algunos parámetros judiciales y parlamentarios, aún incipiente en su proceso de adecuación. Cabe acotar que posee un Instituto de Políticas Públicas en Derechos Humanos que trata de informar y fomentar los procesos de memoria, justicia y reparación a las víctimas de las violaciones y atropellos durante las dictaduras militares.

\subsection{Alianza Bolivariana para los pueblos de nuestra América - Tratado de Comercio de los Pueblos (ALBA-TEP) ${ }^{6}$}

A diferencia de otros enfoques de integración, el ALBA no nace como una propuesta propia, sino como reacción frente al Área de Libre Comercio de las Américas (ALCA). En la III Cumbre de las Américas, en Quebec, Canadá, en abril de 2001, Venezuela manifestó no suscribir el ALCA. En diciembre de 2001, en el marco de la III Cumbre de Jefes de Estado y de Gobierno de la Asociación de Estados del Caribe, desarrollada en la isla de Margarita, Venezuela, tanto Cuba como el país anfitrión proponen una organización que pueda aglutinar a los Estados de la región y formular una "integración integral, económica, social, política y cultural". Finalmente, el 14 de diciembre de 2004 Venezuela y Cuba suscriben en La Habana el acta fundacional y sus protocolos de inicio. El 29 de abril de 2006 Bolivia se adhiere, proponiendo que se incorporen "Tratados de Comercio de los Pueblos" para promover el "intercambio solidario y complementario" y frenar a los Tratados de Libre Comercio que a juicio del Presidente boliviano "persiguen incrementar el poder y el dominio de los transnacionales".

Nicaragua se suma al ALBA el 11 de enero de 2007. El 26 de enero de 2008 ingresa Dominica y el 25 de agosto del mismo año lo hace Honduras. El 24 de junio de 2009 Ecuador se adhiere, sumado con San Vicente y Las Granadinas y Antigua y Barbuda. El 30 de julio de 2013 se unía Santa Lucía y posteriormente el 14 de diciembre de 2014 lo consiguieron San Cristóbal y Nieves y Granada. Cabe acotar que Honduras abandonó la instancia durante el gobierno transitorio del presidente Roberto Micheletti.

El ALBA ha tenido trece Cumbres ordinarias, ocho extraordinarias y dos en conjunto con PETROCARIBE. Aunque se estructura en áreas, la dinámica

\footnotetext{
${ }^{6}$ Véase www.alba-tcp.org/
} 
es altamente centralizada y carece de estructuras parlamentarias y judiciales. La óptica por la que se trabaja es en proyectos "Grannacionales" que tiene como meta la construcción de iniciativas supranacionales y una vertiente económica de solidaridad. En lo ideológico el fundamento "...viene dado por la afinidad conceptual de quienes integramos el ALBA, en cuanto a la condición crítica acerca de la globalización neoliberal y la necesidad de romper el esquema del comercio basado en la ficción del libre mercado". En el esquema de trabajo propone empresas conjuntas y proyectos mancomunados, así como propuestas de bancos e integración financiera. Los países que lo integran poseen cerca de setenta millones de habitantes, de los cuales el 64\% es población urbana, y el $66 \%$ de sus exportaciones son combustibles minerales, lubricantes y materiales relacionados. A diferencia de lo que son el MERCOSUR y la Alianza del Pacífico, la unión creada en torno al ALBA es marcadamente política, sustentando su acercamiento, no en una perspectiva financiera y económica, pues su estructura interna no se parece, sino en incrementar vínculos ideológicos y de cercanía en el ejercicio del poder. La gran mayoría de gobernantes del mecanismo, provienen de partidos políticos que no son tradicionales en sus países (Partido Socialista Unido de Venezuela, Alianza País de Ecuador o Movimiento Al Socialismo en Bolivia) o son de organizaciones que tienen un marcado acento antiestadounidense.

\subsection{Alianza del Pacífico ${ }^{7}$}

Es un mecanismo constituido el 28 de abril de 2011 y creado jurídicamente en Paranal, Antofagasta, Chile, el 6 de junio del año siguiente, en el que Colombia, Chile, México y Perú buscaban desarrollar modelos innovadores que les permitieran erigirse como una opción competitiva desde diversos ámbitos. Además de los miembros fundadores, Costa Rica y Panamá manifestaron su intención de sumarse a la organización, adelantando diversos acuerdos que en el corto plazo deben llevarlos a pertenecer a la estructura formal.

La Declaración de Lima, suscrita el 28 de abril de 2011 por los presidentes de Chile, Colombia y México, además de un enviado especial de Panamá, parte de una serie de consideraciones en las que los asistentes manifiestan coincidir en los avances en materia de desarrollo que se tradujo en el crecimiento del empleo, el fortalecimiento de la democracia y la reducción de la pobreza.

Sumado a ello, el esquema indica que reconoce los acuerdos de libre comercio entre los Estados participantes y establece que la meta no es sólo el fortalecimiento del Arco del Pacífico Latinoamericano, sino (contrario a lo que

${ }^{7}$ Véase www.alianzapacifico.net/ 
normalmente se señala), una mayor proyección hacia el continente asiático. Es decir que se busca un espacio que trascienda la regionalización e incluso puedan superarse otras experiencias como la del Foro de Cooperación Económica Asia - Pacífico (del que forman parte todos los miembros de la Alianza del Pacífico, salvo Colombia) y que pese a funcionar desde 1989, no posee un tratado formal que le otorgue institucionalidad.

Sobre las prioridades iniciales que otorga la Declaración de Lima a la incipiente organización, se observa que el énfasis se hará en facilitar el flujo migratorio, cooperación en materia de seguridad y financiera, capacidad de inversión conjunta y fortalecimiento de equipos técnicos. El Acuerdo suscrito en su IV Cumbre en Paranal, el 6 de junio de 2012, establece que la organización es un "área de integración regional", cuyos miembros reivindican la democracia, respetan la separación de poderes y ejercen la defensa de los derechos humanos.

Aunque los exhortos democráticos inician el documento, es notable el marcado acento comercial y económico que tiene. Incluso, es pertinente señalar que se reconocen los deberes de Colombia y Perú como Estados miembro de la Comunidad Andina de Naciones. Al revisar los objetivos de la Alianza del Pacífico se observa que las metas se conseguirán al avanzar de forma paulatina hacia la libre circulación de bienes, servicios, capitales y personas, al impulsar un mayor crecimiento, desarrollo y competitividad de las economías de los Estados miembro, para disminuir la inequidad, superar la exclusión y vencer la pobreza, y buscar la promulgación de políticas el Pacífico asiático. El acuerdo establece que para alcanzar las metas se recurrirá a mecanismos de fomento a zonas de libre comercio, a incentivar la libre circulación de capitales, a simplificar los trámites aduaneros y a facilitar los trámites consulares.

Las instancias de organización recaen en un Consejo de Ministros (de comercio y relaciones exteriores) y en una Presidencia pro témpore en la que cada país durará un año en el ejercicio. Para que un Estado pueda sumarse a la estructura, debe haber aprobación unánime en el Consejo de Ministros y previamente, el país que ha manifestado su voluntad de ingresar, tiene que tener tratados de libre comercio con todos los miembros.

A la par de haber suscrito el Acuerdo Marco, en la IV Cumbre se formuló una declaración en la que se reconoce haber alcanzado grandes logros en lo relativo a la promoción de las inversiones, cambio climático y comercio electrónico, además de seguir intentando eliminar aranceles comunes, crear una certificación electrónica de origen y establecer un mecanismo de movilidad académica y estudiantil para cursantes de pregrado y postgrado.

Aprovechando la participación en la XXII Cumbre Iberoamericana de Jefes de Estado y de Gobierno celebrada en Cádiz, España, se congregaron el 17 de 
noviembre de 2012 los Presidentes de Perú, Chile, México y Colombia para ratificar la necesidad de proyectar los objetivos hacia el Pacífico y enumerar los logros que se habían alcanzado hasta el momento, fundamentalmente en lo referente a eliminación de aranceles, supresión de visados y movilidad de personas. Hubo dos hechos que merecen mención porque pueden indicar la idea de la Alianza del Pacífico por trascender. El primero, es la creación de un Consejo Empresarial para vincular la iniciativa privada y el segundo, el ingreso de España, Uruguay, Australia, Canadá y Nueva Zelanda como observadores. La idea de vincular al empresariado se concretó con una cumbre en Cali, Colombia, se habló inclusive de integrar representaciones diplomáticas, asumiendo experimentalmente una Embajada compartida en Ghana y un espacio que Perú y Colombia mantienen en Vietnam y otro que Colombia y Chile poseen en Argelia y Marruecos. Esto es importante porque pareciera ir migrándose de una visión estrictamente económica a una integración en otros ámbitos que implique mecanismos de lucha contra la corrupción, desarrollo científico e investigativo y la posibilidad de institucionalizar la "Visa Alianza Pacífico".

El 10 de febrero de 2014 la institucionalización de la Alianza del Pacífico se vio fortalecida con la firma en Cartagena de Indias, Colombia, del Protocolo Adicional al Acuerdo Marco en el que se formaliza e indica la sistematización a seguir para poder alcanzar los elementos de integración, partiendo de la especificación de los organismos responsables por cada país y de establecer un conjunto de definiciones que engloban y uniforman a los cuatro Estados fundadores. De hecho, en una declaración adicional, los mandatarios manifestaron que con el protocolo adicional se culminaba una etapa trascendental en la integración regional y que se fortalecerán los vínculos entre los países al concretarse el proceso de liberalización de bienes, servicios e inversiones. Hay también éxitos referentes a materia educativa, investigativa e incentivos a la innovación, culminando la declaración con un documento anexo que sienta las metas inmediatas que deben llevarse a cabo.

\section{ANÁLISIS ESTADÍSTICO DE INDICADORES ALTERNATIVOS DE DESARROLLO COMO EJES DE INTEGRACIÓN REGIONAL}

En un mundo globalizado, los países que hacen parte de América Latina no pueden actuar como agentes independientes del resto del mundo. Desde el enfoque multidimensional del desarrollo, deben crear condiciones que favorezcan, y amplíen oportunidades y capacidades para que la ciudadanía promueva resultados que permitan el desarrollo humano. Para el logro de estos objetivos, los esquemas de integración regional se muestran como mecanismo útiles para favorecerlos. 
Los países que integran América Latina han profundizado en los últimos años lazos de integración en la conformación de diversos grupos, pero ésta integración debe ser entendida como algo más que una agregación de entidades, por el contrario debe lograrse una fusión densa y profunda, una unificación de potencialidades y fortalezas para el desarrollo de sus ciudadanos (Díaz, 2012). En este sentido, los países de la región buscan favorecer sus políticas públicas integrándose para dar respuestas a las demandas sociales y mejorar la calidad de vida de sus habitantes. En virtud de lo anterior, el propósito del análisis es determinar si la gobernabilidad para desarrollos integrales en la región se puede estudiar a partir de la conformación de alianzas que tienen como objetivo integrase alrededor de fines políticos y/o económicos principalmente.

La sub-región de América Latina no es homogénea, a pesar de tener vínculos geográficos, históricos y culturales, cada país aplica sus propias políticas para enfrentar sus problemas; pero a pesar de ello, existen similitudes significativas en algunos países (Levy-Carciente, Phélan y Perdomo, 2014). Las alianzas conformadas en la sub-región buscan unificar esfuerzos con similares objetivos en común y en primera instancia pareciera que quienes lo conforman tienen elementos que los hacen muy homogéneos. A pesar de que la conformación de alianzas atiende de forma a expresa a intereses diferentes -como puede ser de orden económico y otros de orden más político- la intención del estudio es analizar si en las agrupaciones y los países que la conforman, manifiestan de forma similar las capacidades y oportunidades de sus poblaciones. En este sentido, se formula como hipótesis, que los integrantes de Mercado Común del Sur, la Alianza del Pacifico y la Alianza Bolivariana para los Pueblos de América, tengan condiciones y/o resultados en políticas públicas homogéneos internamente a los grupos.

Se realizará un análisis a diecinueve países de Latinoamérica, haciendo uso de grupos compuestos por los tres acuerdos de integración descritos anteriormente: MERCOSUR, AP y ALBA. El objetivo es determinar cuáles son las características que permiten diferenciar los acuerdos y detectar posibles adhesiones, por similitud de características a partir de indicadores alternativos al desarrollo.

El análisis del grado de homogeneidad e integración de la región, se puede realizar mediante la aplicación de dos procedimientos estadísticas: Análisis de Conglomerados y Análisis Discriminante. El primero tiene como objetivo determinar los países que estadísticamente conforman grupos homogéneos de acuerdo a ciertas características significativas que tienen en común, medidas a partir de algún indicador. En el segundo procedimiento, Análisis Discriminante, se conocen a priori los grupos de países, y lo que se busca es determinar las características que significativamente los diferencia como grupos. 
Justificado en lo descrito y asociado al objetivo de estudio, la técnica estadística más apropiada para éste estudio es el Análisis Discriminante, técnica que busca una función lineal de modo que maximice la variabilidad entre los grupos para discriminarlos y determinar si existen diferencias significativas entre un conjunto de variables de dos o más grupos, así como identificar que variables tienen mayor poder discriminatorio.

El Análisis Discriminante se realiza con una selección de índices de bienestar, de libertades civiles, aspectos políticos, económicos y ambientales que conforman un perfil multidimensional de cada uno de los países y de los respectivos bloques o asociaciones, con una perspectiva alternativa al desarrollo. La selección de los indicadores se basó en primer lugar en una alternativa a los indicadores tradicionales de relaciones comerciales para estudiar convenios político-económicos, tales como importaciones y exportaciones, preferencias arancelarias y cuotas, para ampliar a un compendio de indicadores más relacionados al desarrollo integral de los países que componen los bloques.

En este sentido, para la selección de los índices, como primer elemento de análisis, desde hace varios años, se ha realizado una copiosa recopilación documental y electrónica de índices alternativos sobre diversos temas asociados al desarrollo. Se tomaron índices del inventario de Romina Bandura (Bandura, 2008), índices sobre derechos civiles y políticos de la base de datos de Pipa Norris ${ }^{8}$, de informes y documentos internacionales como los Informe de Desarrollo Humano, y fundamentalmente a través de búsquedas propias mediante fuentes electrónicas. Resultado de este esfuerzo se han logrado identificar sesenta índices que miden, de una u otra forma, aspectos ambientales, socio-económicos, políticos, tecnológicos, institucionales. Del total de índices recolectados se seleccionaron en primera instancia aquellos que contaban con datos completos para todos los países latinoamericanos. En segunda instancia, para los índices seleccionados se realizó un análisis comparativo de las dimensiones que poseían, con el objetivo de reducir la presencia multiplicada de la misma dimensión y evitar algún tipo de covarianzas entre los indicadores seleccionados ${ }^{9}$. En virtud de lo anterior se seleccionaron 10 índices $\left(\right.$ Tabla \#1) ${ }^{10}$,

${ }^{8}$ Véase http://www.pippanorris.com

${ }^{9}$ Tal es el caso del Îndice de Libertad de Prensa que está incorporado en el Índice de Calidad Institucional, Índice de Progreso Social y el Índice de Estado Frágil.

${ }^{10}$ Véase: http://www.fundacionlibertad.org.pa/;

http://internationalpropertyrightsindex.org/;

http://www.eiu.com/public/topical_report.aspx?campaignid=Wcol2014; http://www.eiu.com/public/topical_report.aspx?campaignid=Democracy0814; http://atlas.media.mit.edu/en/; http://www.cato.org/blog/world-misery-index-108countries\#3yFpFC:Xnx; 
Tabla \#1. Índices. Países de América Latina

\begin{tabular}{l|c|c|c|c|c|c|c|c|c|c}
\hline Países/Índices & $\begin{array}{c}\text { Calidad } \\
\text { Institucional } \\
\mathbf{( 2 0 1 1 )}\end{array}$ & $\begin{array}{c}\text { Índice de } \\
\text { Derecho de } \\
\text { Propiedad } \\
\mathbf{( 2 0 1 4 )}\end{array}$ & $\begin{array}{c}\text { Índice de } \\
\text { Costo de } \\
\text { Vida (2014) }\end{array}$ & $\begin{array}{c}\text { Indice de } \\
\text { Democracia } \\
\mathbf{2 0 1 3}\end{array}$ & $\begin{array}{c}\text { Índice de } \\
\text { Complejidad } \\
\mathbf{( 2 0 1 2 )}\end{array}$ & $\begin{array}{c}\text { Índice de } \\
\text { Aflicción } \\
\mathbf{( 2 0 1 4 )}\end{array}$ & $\begin{array}{c}\text { Índice de } \\
\text { Progreso } \\
\text { Social (2015) }\end{array}$ & $\begin{array}{c}\text { Índice de } \\
\text { Innovación } \\
\text { Global (2014) }\end{array}$ & $\begin{array}{c}\text { Índice de } \\
\text { Globalización } \\
\text { (2013) }\end{array}$ & $\begin{array}{c}\text { Índice de } \\
\text { Estado Frágil } \\
\text { (2014) }\end{array}$ \\
\hline Argentina & 0,372 & 4,40 & 67,12 & 6,84 & 0,304 & 68,00 & 73,08 & 35,13 & 58,30 & 47,30 \\
\hline Bolivia & 0,298 & 4,50 & 40,02 & 5,79 & $-0,335$ & 18,38 & 63,36 & 27,76 & 53,08 & 78,90 \\
\hline Brasil & 0,482 & 5,50 & 55,33 & 7,12 & 0,316 & 42,79 & 70,89 & 36,39 & 59,21 & 61,40 \\
\hline Chile & 0,833 & 6,80 & 53,21 & 7,8 & $-0,029$ & 14,42 & 78,29 & 40,64 & 72,91 & 42,00 \\
\hline Colombia & 0,502 & 5,00 & 38,92 & 6,55 & 0,412 & 19,61 & 68,85 & 35,5 & 52,40 & 83,10 \\
\hline Costa Rica & 0,688 & 6,00 & 62,53 & 8,03 & 0,362 & 26,87 & 77,88 & 37,3 & 61,64 & 48,50 \\
\hline Cuba & 0,134 &.. &.. & 3,52 &.. &.. & 60,83 &.. & 48,88 & 70,80 \\
\hline Ecuador & 0,282 & 5,00 & 45,57 & 5,87 & $-0,420$ & 13,72 & 68,25 & 27,5 & 54,01 & 77,30 \\
\hline El Salvador & 0,559 & 4,90 & 50,64 & 6,53 & 0,186 & 12,56 & 64,31 & 29,08 & 62,59 & 72,00 \\
\hline Guatemala & 0,427 & 4,80 & 50,42 & 5,81 & $-0,154$ & 21,21 & 62,19 & 30,75 & 59,67 & 80,30 \\
\hline Honduras & 0,372 & 4,50 & 53,06 & 5,84 & $-0,300$ & 28,07 & 61,44 & 26,73 & 60,93 & 77,90 \\
\hline México & 0,534 & 5,20 & 41,26 & 6,91 & 0,989 & 10,05 & 67,50 & 36,02 & 59,25 & 71,10 \\
\hline Nicaragua & 0,365 & 4,60 & 47,85 & 5,46 & $-0,790$ & 25,06 & 62,20 & 25,47 & 54,42 & 78,40 \\
\hline Panamá & 0,626 & 5,60 & 55,16 & 7,08 & 0,449 & 9,39 & 71,79 & 38,3 & 67,43 & 55,70 \\
\hline Paraguay & 0,328 & 4,10 &.. & 6,26 & $-0,329$ & 27,01 & 67,10 & 31,59 & 57,57 & 71,60 \\
\hline Perú & 0,589 & 5,00 & 49,43 & 6,54 & $-0,182$ & 20,09 & 67,23 & 34,73 & 64,30 & 72,90 \\
\hline R. Dominicana & 0,434 & 4,90 & 55,95 & 6,74 & $-0,021$ & 27,98 & 62,47 & 32,29 & 60,22 & 73,40 \\
\hline Uruguay & 0,677 & 6,10 & 68,55 & 8,17 & 0,332 & 28,37 & 79,21 & 34,76 & 65,28 & 37,90 \\
\hline Venezuela & 0,099 & 3,20 & 111,01 & 5,07 & $-0,692$ & 106,30 & 63,45 & 25,66 & 49,44 & 76,50 \\
\hline
\end{tabular}

Fuente: Páginas oficiales de los distintos indicadores señalados

Calidad Institucional, Derecho de Propiedad, Costo de Vida, Democracia, Complejidad, Aflicción, Progreso Social, Innovación Global, Globalización y Estado Frágil; todos ellos multidimensionales.

Una vez escogidos los 10 índices, se cotejaron con las dimensiones trabajadas en documentos previos y que conforman el enfoque adoptado para medir el bienestar (Levy-Carciente, Phélan y Perdomo, 2015). En buena medida también asociados con las dimensiones establecidas para las metas de desarrollo sostenible de la ONU (2015) para el año 2030, relacionados a los aspectos económicos, sociales, ambientales, de violencia, cohesión social, innovación, institucionalidad, transparencia y la integración; dimensiones rescatados y recomendados por el Panel de Alto Nivel de la ONU (2013) como factores primordiales y que ahora serán monitoreadas para las metas de los próximos 15 años en los objetivos de cada país de las Naciones Unidas y en sus integraciones regionales.

En el presente ejercicio se presentan y analizan los 10 índices seleccionados en dos grandes dimensiones que explican, en cierta medida, la multidimensionalidad del concepto de desarrollo y sus relaciones con el bienestar y la calidad

http://www.socialprogressimperative.org/es; http://www.globalinnovationindex.org/; http://www.kof.ethz.ch/;

http://library.fundforpeace.org/library/cfsir1423-fragilestatesindex2014-06d.pdf 
Figura \#1. Potencialidades y Eficiencia Sinérgica

\begin{tabular}{|c|c|c|c|c|c|c|c|c|c|c|c|c|c|}
\hline \multirow[b]{3}{*}{ Indices/Dimensiones } & \multicolumn{9}{|c|}{ Potencialidades } & \multirow{2}{*}{\multicolumn{4}{|c|}{ Efficiencia Sinérgica }} \\
\hline & \multicolumn{2}{|c|}{ Capacidades Humanas } & \multirow{2}{*}{$\begin{array}{c}\begin{array}{c}\text { Capacidades } \\
\text { Naturales }\end{array} \\
\text { Ambiente }\end{array}$} & \multicolumn{6}{|c|}{ Oportunidades } & & & & \\
\hline & $\begin{array}{l}\text { Salud, } \\
\text { Educacionn, } \\
\text { Ingreso }\end{array}$ & $\begin{array}{c}\text { Equidad } \\
\text { Distributiva }\end{array}$ & & Politicas & \begin{tabular}{|c} 
Agencia \\
(Expresiou de \\
Iddas)
\end{tabular} & $\begin{array}{l}\text { Satisfacción } \\
\text { Material }\end{array}$ & \begin{tabular}{|l} 
Respeto a la \\
Diferencia
\end{tabular} & Justicia & Derechos & Sociedad & Tecnologia & Ecológica & Relacional \\
\hline Calidad Institucional & & & & & $x$ & $x$ & & $x$ & & & & & \\
\hline $\begin{array}{l}\text { İdice de Derecho de } \\
\text { Propiedad }\end{array}$ & & & & & & & & $\mathrm{x}$ & $x$ & $x$ & & & \\
\hline Índice de Costo de Vida & & & & & & $x$ & & & & & & & \\
\hline Indice de Democracia & & & & $x$ & & & $\mathrm{x}$ & & & & & & \\
\hline Índice de Complejidad & & & & & & & & & & & $\mathrm{x}$ & & \\
\hline Índice de Aflicción & $x$ & & & & & & & & & & & & \\
\hline Índice de Progreso Social & $x$ & & $x$ & & $x$ & & $x$ & $x$ & $x$ & & & $x$ & \\
\hline $\begin{array}{l}\text { İndice de Innovación } \\
\text { Global }\end{array}$ & $x$ & & $x$ & $x$ & & & $\mathrm{x}$ & & & & $x$ & $x$ & \\
\hline Índice de Globalización & $x$ & & & & & & & & & & & & $\mathrm{x}$ \\
\hline
\end{tabular}

de vida de las poblaciones (ver Figura \#1). De las dos dimensiones que se consideran, la primera se refiere a las Potencialidades (condiciones básicas necesarias); mientras la segunda se refiere a las derivaciones de estas potencialidades en Eficiencia Sinérgica, relacionados con la calidad de vida que disfrutan las personas en todos los países. Las potencialidades se dividen a su vez en dos categorías: aquellas mediciones que recogen las capacidades -humanas y naturales- con las que cuenta una población y aquéllas que recogen las oportunidades que el ordenamiento institucional ofrece a dicha sociedad. De otro lado, la eficiencia y sostenibilidad de apropiadas condiciones de vida para la sociedad, dependerá de la capacidad de alcanzar una combinación sinérgica de las potencialidades. Esto es lo que hemos definido como eficiencia sinérgica (Levy et al, 2015).

Las capacidades fueron evaluadas a partir de derechos y aspectos relacionados con la salud, educación, los ingresos, la desigualdad y el ambiente. Las oportunidades fueron evaluadas a partir de las condiciones institucionales: las reglas de juego imperantes (que incluye leyes, normas, reglas de conducta, ordenamientos organizativos, y otros) y para ello se consideraron: la libertad de acción política, la libertad de expresión de ideas, la libertad para satisfacer las necesidades materiales, el respeto a la diferencia, a la alteridad y el respeto a las reglas y normas establecidas. Por su parte la eficiencia sinérgica fue evaluada a partir de aspectos como satisfacción colectiva, avances tecnológicos, sostenibilidad ambiental y relacionamiento mundial.

Con la selección de indicadores de la Tabla \#1, los países que conforman la sub-región y la técnica de Análisis Discriminante, se aplica una serie de pruebas estadísticas para determinar el comportamiento de los grupos de América 
Latina. Es importante resaltar que estos grupos conformados no son el resultado de un proceso aleatorio o natural, son en nuestro caso agrupaciones existentes, conformadas por voluntad política.

Dado que el análisis discriminante exige que las agrupaciones conformen grupos disjuntos entre sí, se realizaron dos pruebas estadísticas:

- Prueba I: considera los grupos ALBA (Venezuela, Cuba, Nicaragua, Ecuador y Bolivia), AP (Colombia, México, Perú, Chile, Costa Rica y Panamá, los dos últimos en proceso de anexión), y un tercero con el resto de países bajo estudio no asociados a estos esquemas de integración (Brasil, República Dominicana, Uruguay, Paraguay, Argentina, Honduras, Guatemala, El Salvador);

- Prueba II: analiza MERCOSUR (Argentina, Brasil, Paraguay, Uruguay y Venezuela), AP (Colombia, México, Perú, Chile, Costa Rica y Panamá, los dos últimos en proceso de anexión) y un tercero con el resto de países bajo estudio no asociados a estos esquemas de integración (Bolivia, Cuba, Ecuador, El Salvador, Guatemala, Honduras, Nicaragua y República Dominicana).

La conformación de un tercer grupo para el resto de países de Latinoamérica asociados a los esquemas de integración revisados en cada prueba atiende a la necesidad de analizar toda la región en su conjunto y considerar la cercanía que estos países pudieran tener con las agrupaciones existentes. La consideración de un país en un grupo dependerá de ejercicio que se esté analizando, en la Tabla \#2 se puede observar los países que conforman los grupos en cada prueba estadística.

Es importante resaltar que otros países del Caribe que forman parte de la región y/o pertenecen algún grupo, como el caso del resto de los países miembros del ALBA, no se integraron al análisis. Solo se incluirá República Dominicana y Cuba como islas de habla hispana, el resto de las islas tienen dependencia de otros países que pueden distorsionar el análisis, tal es el caso de Puerto Rico como país dependiente de los Estados Unidos y otras islas de Caribe dependientes de Inglaterra, Holanda y Francia, donde adicionalmente presentan un acercamiento cultural y lingüístico muchos más asociados a ellos. Además, en algunos casos, estos países presentan ausencia de datos para diversos indicadores que podrían comprometer la consistencia del estudio, como es el caso de algunos países miembros del ALBA.

En el caso particular de Cuba, a pesar de tener valores ausentes, se consideró incluir en el estudio dado su particularidad e importancia para los análisis de la sub-región, éste país tiene ausencia de datos para cinco de los índices 
Tabla \#2. Conformación de Grupos según prueba estadística

\begin{tabular}{|c|c|c|}
\hline Países/Pruebas & $\begin{array}{c}\text { Prueba Estadística I: } \\
\text { Grupo ALBA =1, } \\
\text { Grupo AP }=\mathbf{2} \text { y } \\
\text { Otros }=3\end{array}$ & $\begin{array}{c}\text { Prueba Estadística II: } \\
\text { Grupo MERCOSUR =1, } \\
\text { Grupo AP }=\mathbf{2} \text { y } \\
\text { Otros }=\mathbf{3}\end{array}$ \\
\hline Argentina & 3 & 1 \\
\hline Bolivia & 1 & 3 \\
\hline Brasil & 3 & 1 \\
\hline Chile & 2 & 2 \\
\hline Colombia & 2 & 2 \\
\hline Costa Rica & 2 & 2 \\
\hline Cuba & 1 & 3 \\
\hline Ecuador & 1 & 3 \\
\hline EI Salvador & 3 & 3 \\
\hline Guatemala & 3 & 3 \\
\hline Honduras & 3 & 3 \\
\hline México & 2 & 2 \\
\hline Nicaragua & 1 & 3 \\
\hline Panamá & 2 & 2 \\
\hline Paraguay & 3 & 1 \\
\hline Perú & 2 & 2 \\
\hline R. Dominicana & 3 & 3 \\
\hline Uruguay & 3 & 1 \\
\hline Venezuela & 1 & 1 \\
\hline
\end{tabular}

seleccionados, por lo que se imputaron dichos valores para poder incluirlo en el análisis. La técnica de imputación de los datos aplicada, dado la particularidad de los indicadores con presencia de valores extremos, se realizó por medio de la variable más correlacionada donde existe el valor ausente y con dicha variable se tomó el valor adyacente del vecino más próximo por encima y por debajo de Cuba para interpolar dos valores que promediados resultan el valor final imputado, como método más óptimo en este caso. Esta misma técnica se aplicó para imputar el valor ausente que presentaba Paraguay en un indicador (ver Tabla \#3 y detalles en Anexo A).

Para las pruebas se consideraron, en los 10 indicadores seleccionados, los valores más actualizados para los 19 países de la región Latinoamericana (ver 
Tabla \#3. Índices: Los valores ajustados. Países de América Latina

\begin{tabular}{|c|c|c|c|c|c|c|c|c|c|c|c|}
\hline & Índices & $\begin{array}{c}\text { Calidad } \\
\text { Institucional } \\
\quad \text { (2011) }\end{array}$ & $\begin{array}{c}\text { Índice de } \\
\text { Derecho de } \\
\text { Propiedad } \\
\text { (2014) }\end{array}$ & $\begin{array}{c}\text { Índice de } \\
\text { Costo de } \\
\text { Vida (2014) }\end{array}$ & \begin{tabular}{|c|} 
Indice de \\
Democracia \\
2013
\end{tabular} & $\begin{array}{c}\text { Índice de } \\
\text { Complejidad } \\
\text { (2012) }\end{array}$ & $\begin{array}{c}\text { Índice de } \\
\text { Aflicción } \\
\text { (2014) }\end{array}$ & $\begin{array}{c}\text { Índice de } \\
\text { Progreso } \\
\text { Social } \\
(2015)\end{array}$ & $\begin{array}{c}\text { Índice de } \\
\text { Innovación } \\
\text { Global } \\
\text { (2014) }\end{array}$ & $\begin{array}{c}\text { Índice de } \\
\text { Globalización } \\
\text { (2013) }\end{array}$ & $\begin{array}{c}\text { Índice de } \\
\text { Estado } \\
\text { Frágil } \\
\text { (2014) }\end{array}$ \\
\hline Países & Etiqueta & CALIDADIT & IPRI & CLI & IDEMOC & COMPLEJ & MISERY & SPI & ING & GLOBALIZ & FRAGIL \\
\hline Argentina & Ar & 0,372 & 4,40 & 32,88 & 6,84 & 0,304 & 32,0 & 73,08 & 35,13 & 58,30 & 52,70 \\
\hline Bolivia & Bo & 0,298 & 4,50 & 59,98 & 5,79 & $-0,335$ & 81,6 & 63,36 & 27,76 & 53,08 & 21,10 \\
\hline Brasil & $\mathrm{Br}$ & 0,482 & 5,50 & 44,67 & 7,12 & 0,316 & 57,2 & 70,89 & 36,39 & 59,21 & 38,60 \\
\hline Chile & Ch & 0,833 & 6,80 & 46,79 & 7,8 & $-0,029$ & 85,6 & 78,29 & 40,64 & 72,91 & 58,00 \\
\hline Colombia & Co & 0,502 & 5,00 & 61,08 & 6,55 & 0,412 & 80,4 & 68,85 & 35,5 & 52,40 & 16,90 \\
\hline Costa Rica & $\mathrm{Cr}$ & 0,688 & 6,00 & 37,47 & 8,03 & 0,362 & 73,1 & 77,88 & 37,3 & 61,64 & 51,50 \\
\hline Cuba & $\mathrm{Cu}$ & 0,134 & 3,34 & 55,78 & 3,52 & $-0,566$ & 16,0 & 60,83 & 23,82 & 48,88 & 29,20 \\
\hline Ecuador & Ec & 0,282 & 5,00 & 54,43 & 5,87 & $-0,420$ & 86,3 & 68,25 & 27,5 & 54,01 & 22,70 \\
\hline El Salvador & $\mathbf{S r}$ & 0,559 & 4,90 & 49,36 & 6,53 & 0,186 & 87,4 & 64,31 & 29,08 & 62,59 & 28,00 \\
\hline Guatemala & Gu & 0,427 & 4,80 & 49,58 & 5,81 & $-0,154$ & 78,8 & 62,19 & 30,75 & 59,67 & 19,70 \\
\hline Honduras & Ho & 0,372 & 4,50 & 46,94 & 5,84 & $-0,300$ & 71,9 & 61,44 & 26,73 & 60,93 & 22,10 \\
\hline México & $\mathbf{M x}$ & 0,534 & 5,20 & 58,74 & 6,91 & 0,989 & 90,0 & 67,50 & 36,02 & 59,25 & 28,90 \\
\hline Nicaragua & $\mathrm{Ni}$ & 0,365 & 4,60 & 52,15 & 5,46 & $-0,790$ & 74,9 & 62,20 & 25,47 & 54,42 & 21,60 \\
\hline Panamá & Pn & 0,626 & 5,60 & 44,84 & 7,08 & 0,449 & 90,6 & 71,79 & 38,3 & 67,43 & 44,30 \\
\hline Paraguay & $\mathrm{Pr}$ & 0,328 & 4,10 & 59,98 & 6,26 & $-0,329$ & 73,0 & 67,10 & 31,59 & 57,57 & 28,40 \\
\hline Perú & $\mathrm{Pe}$ & 0,589 & 5,00 & 50,57 & 6,54 & $-0,182$ & 79,9 & 67,23 & 34,73 & 64,30 & 27,10 \\
\hline R. Dominicana & Do & 0,434 & 4,90 & 44,05 & 6,74 & $-0,021$ & 72,0 & 62,47 & 32,29 & 60,22 & 26,60 \\
\hline Uruguay & Ur & 0,677 & 6,10 & 31,45 & 8,17 & 0,332 & 71,6 & 79,21 & 34,76 & 65,28 & 62,10 \\
\hline Venezuela & $\mathbf{V z}$ & 0,099 & 3,20 & $-11,01$ & 5,07 & $-0,692$ & $-6,3$ & 63,45 & 25,66 & 49,44 & 23,50 \\
\hline
\end{tabular}

Tabla \#3). Es importante señalar que las ediciones de los indicadores varían entre 2011 y 2014, sin embargo estas diferencias, se consideraron poco relevantes, tomando en cuenta que las variaciones en un mismo indicador en el corto plazo son bajas. En cuanto a las diversas escalas presentes en las variables, no fue necesario su ajuste para el Análisis Discriminante, sin embargo, si lo fue el ajustar las direcciones de cada indicador para que queden todos orientados en el mismo sentido. Por esta razón se ajustaron la dirección en forma creciente de los indicadores Índice de Aflicción, Índice de Costo de Vida e Índice de Estado Frágil (Misery Index, Cost of Living Index, Fragile States Index). Esto para garantizar que en el análisis todos los indicadores estén en un sentido positivo de resultados hacia un mayor desarrollo humano (ver Tabla \#3).

\subsection{Resultados}

Para el análisis discriminante en ambas pruebas se aplicó la técnica denominada Paso a Paso ${ }^{11}$. Si bien varios indicadores pueden tener significativamente poder discriminatorio, la idea en un principio es conocer cuál de los indicado-

${ }^{11}$ Esta técnica permite simplificar al máximo posible la función discriminante en las características significativas que son suficientes para discriminar los grupos, de esta forma no hay que incluir todas las características (variables) que tengan poder discriminatorio para construir una función discriminante. 
res son más influyentes a la hora de discriminar a un individuo en un grupo u otro, de modo que la técnica Paso a Paso utiliza solo aquellos indicadores más influyentes, buscando representar una función discriminante con la menor cantidad de variables posibles y que sea igualmente significativa para diferenciar los grupos. Las variables resultantes permiten maximizar las diferencias de los grupos, digamos en un espacio gráfico, alejándose entre ellos lo máximo posible; por el contrario si se agrega otra variable, la función podrá seguir discriminando a los grupos, pero acercándolos más entre ellos (ver Figura \#2). Por esta razón se seleccionan las variables que proporcionen los centros de los grupos más alejados entre sí y más concentrados dentro de sí.

En la Prueba I (ALBA, AP, Otros), de los 10 indicadores considerados, existen 6 cuyas pruebas reflejan significativamente poder discriminatorio para diferenciar los grupos, estos son: Innovación Global, Calidad Institucional, Compleji-

Figura \#2. Distancia de los grupos de acuerdo al indicador

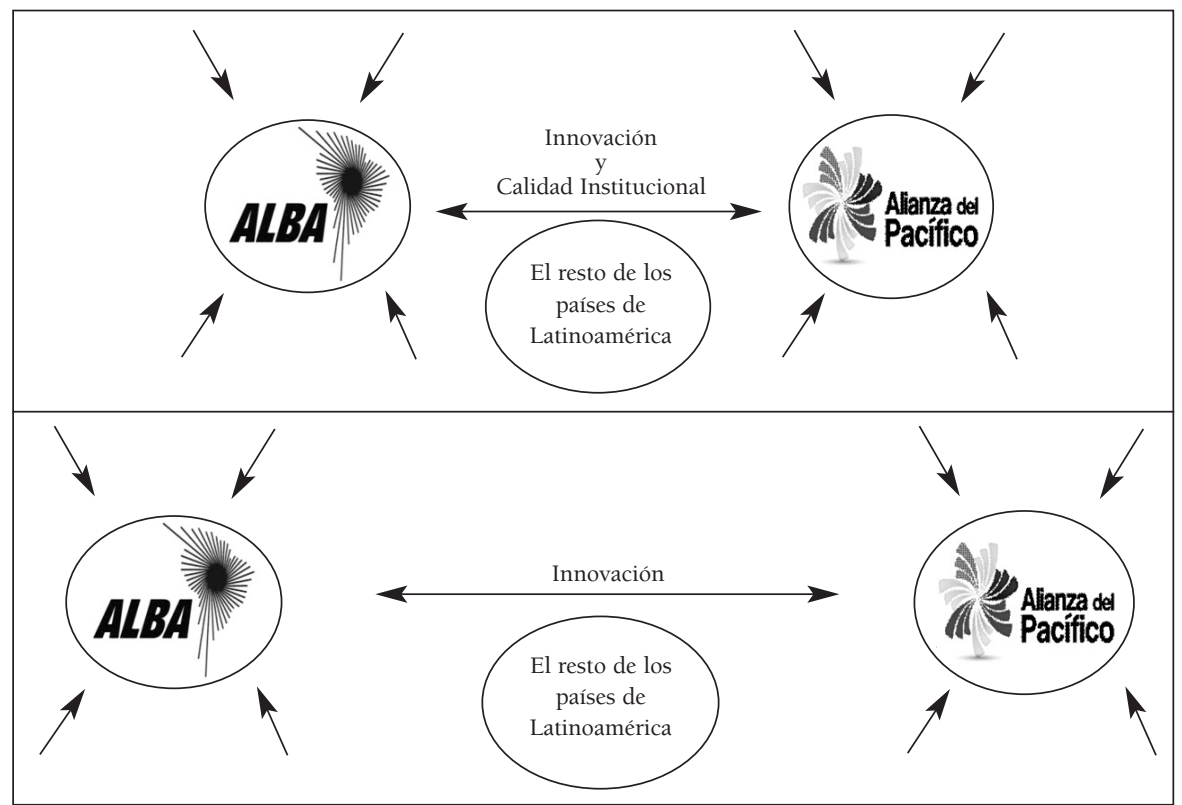

${ }^{12}$ Recoge la pertenencia de los sujetos a los grupos mediante un coeficiente que oscila entre 0 y 1 . Interesa que presente un valor lo más próximo a 1.

${ }^{13}$ Representa la diferencia entre grupos. Los valores próximos a 1 indican similitudes entre grupos, si su valor es cercano a 0 deberá interpretarse que los grupos son diferentes y por lo tanto, la función discriminante puede pronosticar adecuadamente a los sujetos. 
dad Económica, Relacionamiento Global, Democracia y Derecho de Propiedad. Sin embargo, aplicando la técnica Paso a Paso, el resultado es que el indicador de Innovación Global es suficiente para elaborar un modelo discriminante (ver Anexo C), la función explica el $100 \%$ de la variabilidad del modelo, con una correlación canónica ${ }^{12}$ de 0.868 y un valor de Wilks $^{13}$ en 0.246 , indicativos que los grupos son diferentes y que podrán discriminarse adecuadamente con esa variable en una sola función discriminante (ver Anexo D).

En la Figura \#2 se ilustra el caso de la primera prueba estadística, en el que los índices Innovación Global y Calidad Institucional discriminan muy bien a los grupos, pero donde la combinación de ambos hace que se acerquen más entre ellos, mientras que Innovación Global logra por si solo maximizar la distancia entre los grupos y discriminar mucho mejor.

En la Prueba II (MERCOSUR, AP, Otros), de los 10 indicadores considerados, existen 7 cuyas pruebas reflejan significativamente poder discriminatorio para diferenciar los grupos, estos son: Innovación Global, Progreso Social, Calidad Institucional, Complejidad Económica, Democracia, Aflicción y Estado Frágil (ver Anexo B). Una vez aplicada la técnica Paso a Paso, el resultado es que dos funciones discriminantes, la primera con Innovación Global y la segunda con Aflicción, son suficientes para diferenciar los grupos que conforman el análisis y elaborar un modelo discriminante (ver Anexo C), así mismo, las condiciones del modelo resultan adecuadas para discriminar o separar bien a los respectivos grupos en análisis (ver Anexo D).

El hecho de que no todas las variables tengan poder discriminatorio es indicativo de que los grupos en algunas dimensiones no son homogéneos, son países que conforman un grupo con resultados completamente distintos en ese indicador. Por el contrario cuando tienen poder discriminatorio, indica que los grupos deben ser analizados a partir de esas variables, ya que por ser más homogéneos en ese indicador, permiten representar mejor a los países integrados.

Una mejor ilustración de los resultados se obtiene con los valores de los centroides; éstos indican que los grupos son diferentes en la medida que sus distancias medias sean mayores, lo cual permite diferenciar a cada grupo y obtener una medida de su representación espacial (ver Anexo E). En la primera prueba estadística, la representación espacial entre los grupo ALBA, AP y Otros se obtiene con los valores $-2.316 ; 1.922$ y 0.006 respectivamente, sus diferencias son bien marcadas, hacia los extremos, con una distancia entre ALBA y AP que coloca en el centro al resto de los países de Latinoamérica y que además demuestra qué tan diferentes son ambos grupos entre sí. Además, permite observar las posiciones de los países con relación a su grupo de conformación y con relación a los otros grupos (ver Figura\#3). 
Figura \#3. Centroides de los grupos. Prueba I

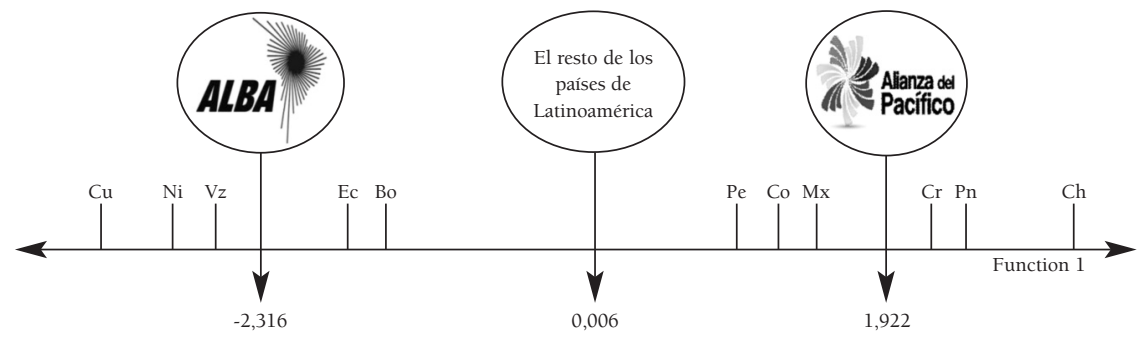

Por su parte en la segunda prueba, también son marcadas las diferencias entre los grupos, especialmente con la segunda función discriminante entre MERCOSUR y AP, aunque con presencia de algunos países muy aislados de sus centroides de grupo y más asociados a otros centroides de otros grupos, lo cual puede significar una asociación mayor hacia otro grupo por compartir características más similares (ver Figura\#4).

Figura \#4. Centroides de los grupos. Prueba II

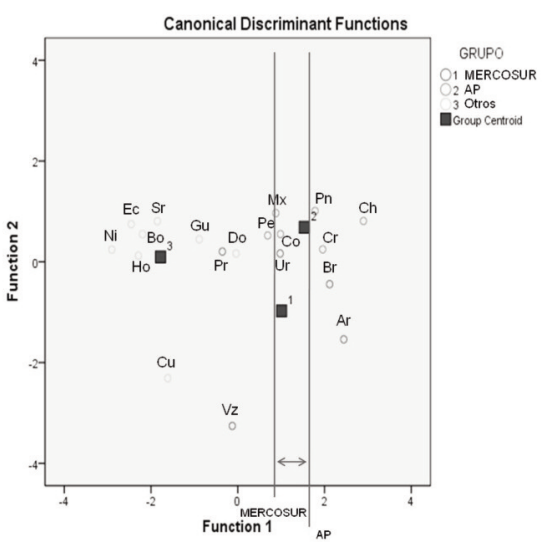

Función 1: Índice de Innovación Global. Se observa que los centroides para MERCOSUR y AP son muy cercanos para esta función, así como bien distantes ambos con el resto de Latinoamérica. Es de notar como particularmente Cuba y Venezuela son los países más alejados de sus respectivos centroides y más cercanos entre sí.

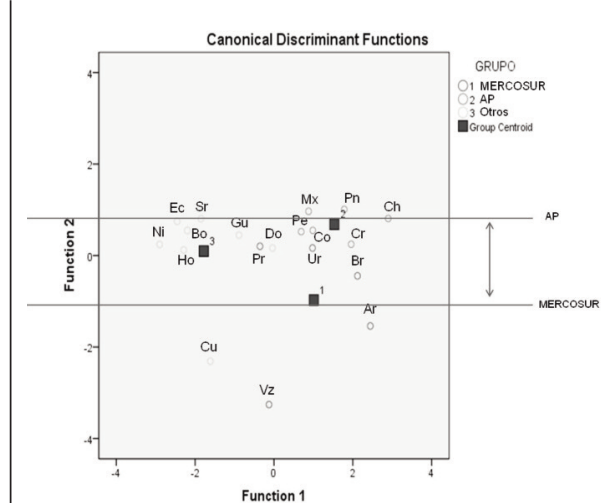

Función 2: Índice de Aflicción. Con esta función se distingue mayores diferencias entre los centroides de los tres grupos, principalmente MERCOSUR y AP. 
Ahora, por ser la Innovación Global un índice con poder discriminatorio para la región en ambas pruebas realizadas y por tratarse de un índice multidimensional, es de interés conocer cuál de sus componentes es el que presenta mayor poder discriminatorio, es decir, aplicar un análisis discriminante en los componentes de éste indicador.

El índice de Innovación Global está compuesto por las dimensiones: Institucionalidad, Capital Humano y de Investigación, Infraestructura, sofisticación del mercado, sofisticación del negocio, salidas de conocimiento y tecnología, y salidas creativas. El resultado obtenido muestra que de las diversas dimensiones que componen este indicador; para países que conforman los grupos de la primera prueba estadística, resulta la Institucionalidad como el factor con mayor poder discriminatorio, y a lo interno de ella se determinó que el ambiente regulatorio y cumplimiento de la ley como las más significativas. Para los países que conforman los grupos de la segunda prueba estadística, el Capital Humano y de Investigación y la Infraestructura son los factores con mayor poder discriminatorio, y a los interno de ellos se determinó que la educación terciaria y sostenibilidad ecológica son las más significativas.

Adicionalmente, es importarte observar la consistencia de los grupos conformados por las alianzas ALBA, MERCOSUR y AP en las dos pruebas realizadas, para esto se aplicó un test estadístico de contraste y probabilidad de pertenencia ${ }^{14}$, cuyo resultado permite obtener la probabilidad estadística de pertenecer al grupo de origen o a otro grupo diferente, dado el perfil del individuo en el indicador de la función discriminante. Para ello se obtiene una Matriz de Confusiones donde se expresan los individuos según su grupo de origen y por el grupo pronosticado al cual tienen un perfil más asociado (ver Tabla \#5 y Anexo F).

De lo anterior se extrae que en la Prueba I, el 100\% de los países del ALBA conservan su pertenencia al grupo, y de los países del grupo 3 (Otros), se observa un país, Honduras, con cercanía al ALBA. Esto quiere decir que Honduras tiene un perfil más asociado a ALBA que a Otros. Por su parte, en el grupo AP, el 83,3\% de los individuos se mantienen como grupo, pero Perú tiene características que lo asocian más hacia el resto de Latinoamérica (Otros) por sus resultados en la función discriminante. Mientras, Brasil y Argentina (ubicados en Otros), tienen un perfil más asociado hacia AP y el resto de los países (Guatemala, El Salvador, Uruguay, Paraguay y República Dominicana) quedan

${ }^{14}$ El cálculo de probabilidad de pertenecer a un grupo, se realiza con la condición de conocer a priori la pertenencia de un individuo a un grupo específico, en nuestro caso ALBA, AP, MERCOSUR, OTROS, para clasificarlo posteriormente al grupo con mayor probabilidad a posteriori. 
Tabla \#5. Resultado de la Clasificación

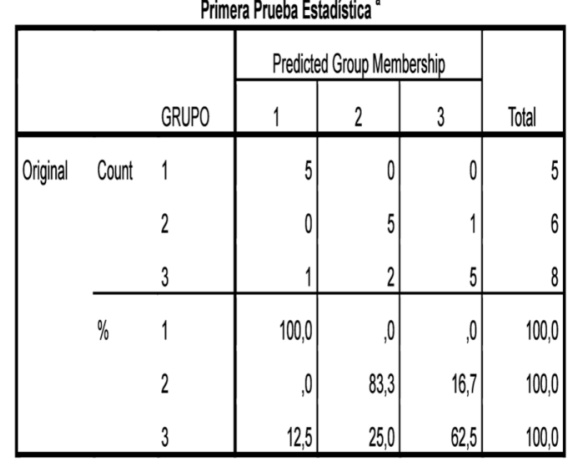

a. $78,9 \%$ of original grouped cases correctly classified.

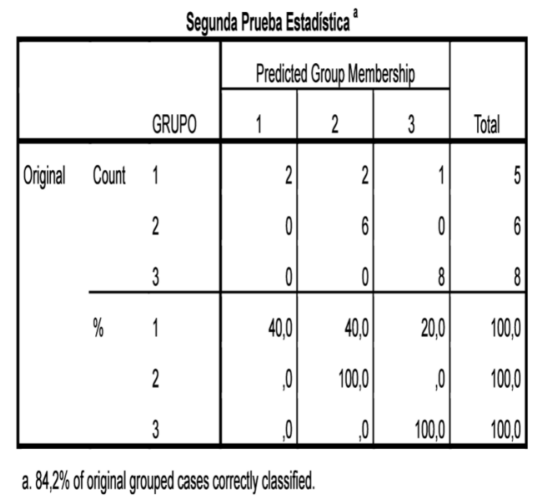

sin cambios de grupo, ya que presentan probabilidades estadísticas no significativas ${ }^{15}$. Sin embargo, los datos permiten observar hacia qué grupo (ALBA o AP) se encuentra más asociado los países ubicados en Otros, así, Guatemala y El Salvador, tiene perfiles más cercanos a los países del ALBA, mientras que Uruguay, Paraguay y República Dominicana se asemejan a los de AP (ver Anexo F).

Para la Prueba II, el 100\% de los países del grupo AP y del grupo de países de Latinoamérica (Otros) conservan su conformación de grupo, mientras que de MERCOSUR tanto Brasil como Uruguay presentan características más similares a la AP, y Paraguay a Otros. Asimismo, se puede observar que del grupo 3 (Otros), Bolivia, Cuba, Ecuador, Honduras y Nicaragua poseen perfiles similares a MERCOSUR, mientras que República Dominicana, Guatemala y El Salvador se muestran más asociados a AP.

Estos resultados indican que MERCOSUR presenta características heterogéneas entre los países que la integran, lo que a largo plazo podría generarle dificultades para la aplicación de políticas comunes. Lo anterior dialoga con planteamientos de Caetano (2009), quien indica que el MERCOSUR requiere un sinceramiento y que ello implica rediscutir su modelo de integración. En cambio, AP y ALBA si bien representan alternativas bien diferenciadas, son grupos más estables por las homogeneidades de los países que la integran, la diferencia entre ellas son las características que las proyectan más o menos hacia el progreso. A pesar de que las diversas agrupaciones analizadas no presentan armonía en todas las dimensiones y que aún entre países que conforman un

${ }^{15}$ Para que la probabilidad sea significativa debe ser mayor a 0,5. 
grupo particular existen diferencias en algunos indicadores, los resultados aconsejan cuales son las áreas donde cada grupo de integración debe hacer más énfasis en sus políticas de integración.

\section{REFLEXIONES FINALES}

La región Latinoamericana es muy heterogénea en cuanto a sus indicadores o mediciones y sucede lo mismo internamente para los diversos grupos de integración conformados. Sin embargo, algunos índices permiten encontrar cierta homogeneidad interna, especialmente el de Innovación Global que aparece como un fuerte discriminador de la integración para América Latina. Este índice es el resultado de la calidad en un entorno institucional político, regulatorio y empresarial estable, de libertades de expresión y acción, con inversión en talento humano, investigación y desarrollo, vínculos de innovación, absorción e impacto del conocimiento, su creación y difusión. De este modo es una medición que refleja las grandes oportunidades para el desarrollo de sus países. La innovación además permite tener una inversión de capital en los países donde se potencia, generando empleo y aumentado el crecimiento económico, que se traducirá en una disminución de la Aflicción, siempre y cuando se mantenga la institucionalidad.

Pero además dentro de los componentes del índice de Innovación Global también se resaltan como determinantes: el ambiente regulatorio, cumplimiento de la ley, la educación terciaria y la sustentabilidad ecológica. Estos resultados no distan de las metas establecidas por la ONU (2015) para el año 2030, donde se debe fomentar la innovación, promover una industrialización sostenible en total equilibrio con la naturaleza y además de tener una infraestructura flexible, transparencia y un estado de derecho para el cumplimiento de las leyes.

El entorno para la innovación es un factor fundamental, tal y como indica Oppenheimer (2014), un mal clima para los negocios, las burocracias, la intolerancia y la corrupción ahuyentaría fácilmente las innovaciones. Además, expresa que en la actualidad el progreso de los países depende cada día menos de los recursos naturales y mucho más de los sistemas educativos, científicos y sus innovaciones, donde los avances científicos determinarán quienes progresan más y quienes se quedarán cada vez más atrás.

Uno de los grandes problemas que ha tenido el Sur de América, es su "baja capacidad para producir bienes con valor agregado y exportar" como consecuencia de un pasado mercantilista y por precariedad en la ciencia y tecnología (Mata, 2011; 125). Por ello, la innovación no es solo determinante para la integración de los países, sino un factor fundamental para su progreso. Las diferencias en los niveles de innovación entre AP y ALBA son bien marcados, no 
tanto en el caso de AP, MERCOSUR, sin embargo en el año 2014, todos los países que integran la AP están en mejores posiciones para hacer negocios que los países que integran el MERCOSUR, los cuales están, en posiciones muy desfavorables según el informe Doing Business 2014 del Banco Mundial (Turzi, 2014).

En este sentido, se debe hacer énfasis en los diversos componentes que integran este índice y no solo a la innovación per se, para adelantar políticas en común desde los diversos grupos de integración alineados al progreso de América Latina; impulsando además lo que Oppenheimer (2014) denomina la globalización de la innovación, donde el proceso para innovar exige de lazos de cooperación entre diversos entes para garantizar un mayor éxito. Los grupos deben hacer grandes esfuerzos para consolidar estrategias de desarrollo, con transferencia de tecnología y formación de talento humano; si la creatividad es un proceso social que genera innovación, entonces los acuerdos de integración tienen su razón de ser en el fomento e intercambio del conocimiento.

La productividad científica correlaciona significativamente con el desarrollo económico y social de una nación, además de darle apertura a una sociedad y formar individuos prósperos (Jaffe et al, 2013). De este modo, las integraciones deben viajar por una vía más allá de acuerdos comerciales y políticos, deben fundamentarse en la cooperación tecnológica y científica para superar las asimetrías existentes, con mecanismos que incrementen el intercambio de conocimiento entre instituciones científicas y tecnológicas (Vessuri y Mercado, 2013).

Así, la innovación científica se nos muestra como la variable que permite sintetizar la capacidad que los países tienen para apalancar su desarrollo. Vale decir que esta innovación tiene como fundamento una sociedad libre en la que el intercambio de ideas sea posible sin cortapisas, y donde los arreglos sociales la fomenten y difundan.

En todos estos resultados se demuestra que la AP es la agrupación mejor posicionada frente a las recomendaciones de la ONU post-2015 para erradicar la pobreza extrema en la tierra para el 2030 y estar alineadas al tercer gran cambio necesario: transformar la economía para empleos y crecimiento inclusivo, aprovechando la innovación y las tecnologías para desatar el dinamismo económico, empleos e inversión (ONU, 2013). Pero tanto la AP como ALBA están sustentados en la perspectiva de quienes las dirigen, solo el tiempo dirá si existirán cambios en las alianzas o será el mismo éxito de las agrupaciones las que permitirá mantenerlas o no en el tiempo (Turzi, 2014), por los momentos, ambos deben dirigir sus políticas alineadas en este sentido para poder encaminarse a la prosperidad. 
Será en la innovación donde se muestren las grandes diferencias de la región: grupos que consoliden mejores estrategias alineadas a la innovación, la institucionalidad y las libertades, tendrán mayor oportunidad para que sus países obtengan resultados más favorables para sus ciudadanos. Por el contrario, grupos con menores avances en esa materia redundará en pobres oportunidades para la ciudadanía. En general la integración de América Latina debe ser amplia e integral y para surgir como región unas de las etapas que debe superar es la dependencia tecnológica y asumir la innovación como eje fundamental para su progreso.

\section{REFERENCIAS}

Álvarez, M; Victoria, M (2011). "Origen y evolución de los parlamentos en los procesos de integración regional: Los casos del Parlamento Europeo y el Parlamento del Mercosur", Colombia Internacional, Vol. 74, pp. 207-229. Retrieved May 14, 2015, from http://www.scielo.org.co/scielo.php?script=sci_arttext\&pid=S0121$56122011000200009 \& \ln g=e n \& t \operatorname{lng}=$ es .

Andrade, M (2011). "El aislamiento de Venezuela de los procesos de integración latinoamericanos", Mundo Nuevo, Año III, Vol. 2 (2011), No 7. Universidad Simón Bolívar. Venezuela.

Bandura, Romina (2008). A Survey of Composite Indices Measuring Country Performance, New York, United State, UNDP/ODS Working Paper, Office of Development Studies, United Nations Development Programme.

Caetano, G (2009) "Integración regional y estrategias de reinserción internacional en América del Sur" Nueva Sociedad, Vol 219 (enero-febrero 2009), ISSN: 0251-3552, www.nuso.org

Consalvi, S.A. (1988). Un Momento Histórico de América Latina, Caracas, Venezuela, Ed. Pomaire.

De Lombaerde, P; Kingah, S; Rettberg, A (2014) "Presentación: Procesos de integración política, social y económica", colomb.int. [online], n.81 (2014), ISSN 0121-5612. [cited 2015-05-14], pp. 19-24. Available from: http://www.scielo.org.co/scielo. php?script=sci_arttext\&pid=S0121-56122014000200003\&lng=en\&nrm=iso.

Díaz, L. (2012). La Integración Latinoamericana [desde 1750 hasta la actualidad], Caracas, Venezuela, G.E. Amerik, Cátedra Latinoamericana.

Jaffe, K; Caicedo, M; Manzanares, M; Gil, M; Rios, A; Florez, A, et al (2013). "Productivity in Physical and Chemical Science Predicts the Future Economic Growth of Developing Countries Better than Other Popular Indices". PLoS ONE 8(6), 2013: e66239. doi:10.1371/journal.pone.0066239.

Levy-Carciente, S; García, A (2007). "La Integración financiera latinoamericana a la luz de la experiencia europea", Revista del BCV, Vol. XXI(1):109-143, 2007, Banco Central de Venezuela. 
Levy-Carciente, Sary; Phélan, Mauricio; Perdomo, Jhoner (2014). "From Progress to Happiness: Measurements for Latin America" Social Change Review, Vol. 12(1) (summer 2014): 73-112. DOI: 10.2478/scr-2014-0004.

Levy-Carciente, Sary; Phélan, Mauricio; Perdomo, Jhoner (2015). "Potencialidades y Sinergias en América Latina", mimeo.

Mata M., L. (2011). Suramérica: Los retos del siglo XXI, Caracas, Venezuela, Ed. Academia Nacional de Ciencias, Fundación Alberto Adriani.

ONU. (2013). Una nueva alianza mundial: erradicar la pobreza y transformar las economías a través del desarrollo sostenible, Nueva York, Estados Unidos, Informe del Grupo de Alto Nivel de Personas Eminentes sobre la Agenda de Desarrollo Post-2015.

ONU. (2015). Indicators and a Monitoring framework for the sustainable development goals. New York, Unites States, A report to the Secretary General of the United Nations by the Leadership Council of the Sustainable Development Solutions Network.

Oppenheimer, A. (2014). ¡Crear o Morir! La esperanza de América Latina y las cinco claves de la innovación. Penguin Randim House, Grupo Editorial. Colombia.

Romero, M. (2005). Venezuela en defensa de la democracial 1958-1998, Caracas, Venezuela, Ed. Fundación para la Cultura Urbana.

Turzi, M (2014). "Asia y la i(des)integración latinoamericana?" Nueva Sociedad, Vol. 250 (marzo-abril 2014), ISSN: 0251-3552, www.nuso.org

Vessuri, H; Mercado, A (2013). "Desarrollo cientifico y tecnológico, políticas tecnológicas e industriales y recursos naturales". Conferencia de la UNASUR sobre recursos naturales y desarrollo integral de la región, (2013), pp. 1-9, UNASUR, Caracas.

\section{ANEXOS}

\section{Anexo A. Imputación de datos}

Para Cuba se imputaron los datos de los indicadores: Índice de Complejidad, Índice de Aflicción, Índice de Costo de Vida, Índice de Derecho de Propiedad, Índice de Innovación Global. Para Paraguay se imputó el dato correspondiente al Índice de Costo de Vida.

A continuación, se ilustra la imputación del dato Índice de Innovación Global para Cuba: La Innovación Global esta correlacionada con la Calidad Institucional. Ambos indicadores dada su relación son útiles para imputar valores ausentes en algún individuo. En nuestro caso Cuba tiene valor ausente en el indicador de Innovación Global, por lo tanto de la información que existe en el indicador de Calidad Institucional se imputara el valor ausente de Cuba en Innovación Global. Cuba se encuentre adyacente entre Venezuela y Ecuador en el indicador de Calidad Institucional, con estos países se obtienen dos valores interpolados para Cuba en la Innovación Global, con los cuales se promedia para obtener un valor final imputado: 


$$
\begin{aligned}
& A=0.134 * \frac{25.66}{0.0994}=34.59 \quad B=0.134 * \frac{27.5}{0.2822}=13.05 \\
& C=A+\frac{B}{2}=23.82
\end{aligned}
$$

\begin{tabular}{l|c|c}
\hline Países/Índices & $\begin{array}{c}\text { Calidad } \\
\text { Institucional } \\
\mathbf{( 2 0 1 1 )}\end{array}$ & $\begin{array}{c}\text { Índice de } \\
\text { Innovación } \\
\text { Global (2014) }\end{array}$ \\
\hline Venezuela & 0,099 & 25,66 \\
\hline Cuba & 0,134 & $\mathrm{C}$ \\
\hline Ecuador & 0,282 & 27,5 \\
\hline Bolivia & 0,298 & 27,76 \\
\hline Paraguay & 0,328 & 31,59 \\
\hline Nicaragua & 0,365 & 25,47 \\
\hline Argentina & 0,372 & 35,13 \\
\hline Honduras & 0,372 & 26,73 \\
\hline Guatemala & 0,427 & 30,75 \\
\hline R. Dominicana & 0,434 & 32,29 \\
\hline Brasil & 0,482 & 36,39 \\
\hline Colombia & 0,502 & 35,5 \\
\hline México & 0,534 & 36,02 \\
\hline El Salvador & 0,559 & 29,08 \\
\hline Perú & 0,589 & 34,73 \\
\hline Panamá & 0,626 & 38,3 \\
\hline Uruguay & 0,677 & 34,76 \\
\hline Costa Rica & 0,688 & 37,3 \\
\hline Chile & 0,833 & 40,64 \\
\hline & & \\
\hline
\end{tabular}

En nuestro estudio se decidió utilizar un promedio para representar el valor final imputado, para evitar el juicio de seleccionar el vecino más próximo por debajo o por encima del individuo a imputar, dado especialmente porque el dato ausente no es el resultado de un proceso aleatorio. Es importante resaltar además que se probaron diversas técnicas de imputación, pero ninguna tenía consistencia al realizar el análisis sin el dato imputado, por lo que esta técnica per- 
mitió que los datos imputados no alteraran el resultado del análisis, es decir el análisis discriminante no se altera por incluir los valores imputados.

\section{Anexo B. Variables con poder discriminatorio}

\section{Tests of Equality of Group Means}

\begin{tabular}{|l|r|r|r|r|r|}
\hline & Wilk' Lambda & \multicolumn{1}{|c|}{ F } & \multicolumn{1}{|c|}{ di1 } & \multicolumn{1}{c|}{ df2 } & \multicolumn{1}{c|}{ Sig. } \\
\hline CALIDADIT &, 338 & 15,644 & 2 & 16 &, 000 \\
COMPLEJ &, 406 & 11,693 & 2 & 16 &, 001 \\
MISERY &, 764 & 2,476 & 2 & 16 &, 116 \\
CLI &, 964 &, 299 & 2 & 16 &, 745 \\
IPRI &, 573 & 5,956 & 2 & 16 &, 012 \\
SPI &, 698 & 3,458 & 2 & 16 &, 056 \\
IDEMOC &, 452 & 9,695 & 2 & 16 &, 002 \\
ING &, 246 & 24,491 & 2 & 16 &, 000 \\
GLOBALL &, 469 & 9,049 & 2 & 16 &, 002 \\
FRAGIL &, 830 & 1,640 & 2 & 16 &, 225 \\
\hline
\end{tabular}

\begin{tabular}{|l|r|r|r|r|r|}
\hline & Wilk' Lambda & \multicolumn{1}{|c|}{$F$} & \multicolumn{1}{c|}{ df1 } & \multicolumn{1}{c|}{ d'2 } & \multicolumn{1}{c|}{ Sig. } \\
\hline CALLDADIT &, 569 & 6,070 & 2 & 16 &, 011 \\
COMPLEJ &, 635 & 4,594 & 2 & 16 &, 027 \\
MISERY &, 677 & 3,822 & 2 & 16 &, 044 \\
CLI &, 710 & 3,260 & 2 & 16 &, 065 \\
IPRI &, 704 & 3,369 & 2 & 16 &, 060 \\
SPI &, 491 & 8,281 & 2 & 16 &, 003 \\
IDEMOC &, 640 & 4,499 & 2 & 16 &, 028 \\
ING &, 342 & 15,394 & 2 & 16 &, 000 \\
GLOBALL &, 790 & 2,122 & 2 & 16 &, 152 \\
FRAGIL &, 681 & 3,744 & 2 & 16 &, 046 \\
\hline
\end{tabular}

Anexo C. Variables con suficiente capacidad explicativa para diferenciar los grupos

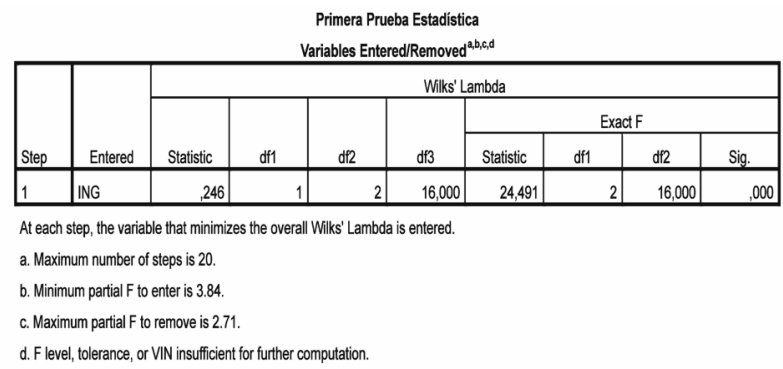

Segunda Prueba Estadistica Variables Entered/Removed ${ }^{\text {a,b,c,d }}$

\begin{tabular}{|c|c|c|c|c|c|c|c|c|c|}
\hline \multirow[b]{3}{*}{ Step } & \multirow[b]{3}{*}{ Entered } & \multicolumn{8}{|c|}{ Wilks' Lambda } \\
\hline & & \multirow[b]{2}{*}{ Statistic } & \multirow[b]{2}{*}{ df1 } & \multirow[b]{2}{*}{$d f_{2}$} & \multirow[b]{2}{*}{$\mathrm{df} 3$} & \multicolumn{4}{|c|}{ Exact $F$} \\
\hline & & & & & & Statistic & df1 & $d \mathfrak{d} 2$ & Sig. \\
\hline 1 & ING & ,342 & 1 & 2 & 16,000 & 15,394 & 2 & 16,000 &, 000 \\
\hline 2 & MISERY & ,179 & 2 & 2 & 16,000 & 10,231 & 4 & 30,000 &, 000 \\
\hline
\end{tabular}

At each step, the variable that minimizes the overall Wilks' Lambda is entered.

a. Maximum number of steps is 20 .

b. Minimum partial $\mathrm{F}$ to enter is 3.84 .

c. Maximum partial $\mathrm{F}$ to remove is 2.71 .

d. F level, tolerance, or VIN insufficient for further computation 
Anexo D. Condiciones para las funciones discriminantes

Segunda Prueba Estadistica

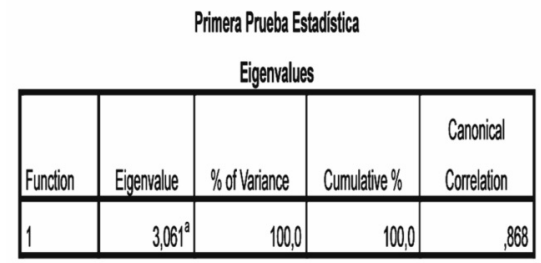

a. First 1 canorical disciminant tunctions were used in the analysis.

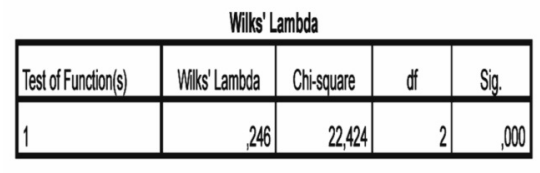

Eigenvalues

\begin{tabular}{|l|r|r|r|r|}
\hline Function & Eigenvalue & \% of Variance & Cumulative \% & \multicolumn{1}{c|}{$\begin{array}{c}\text { Canonical } \\
\text { Correlation }\end{array}$} \\
\hline 1 & $2,784^{\mathrm{a}}$ & 85,4 & 85,4 &, 858 \\
2 &, $477^{\mathrm{a}}$ & 14,6 & 100,0 &, 568 \\
\hline
\end{tabular}

a. First 2 canonical discriminant functions were used in the analysis.

Wilks' Lambda

\begin{tabular}{|l|r|r|r|r|}
\hline Test ofFunction(s) & Wilks' Lambda & Chisquare & \multicolumn{1}{c|}{ df } & \multicolumn{1}{c|}{ Sig. } \\
\hline 1 through2 &, 179 & 26,672 & 4 &, 000 \\
2 & 677 & 6,046 & 1 &, 014 \\
\hline
\end{tabular}

Anexo E. Coeficientes de la función y centroides

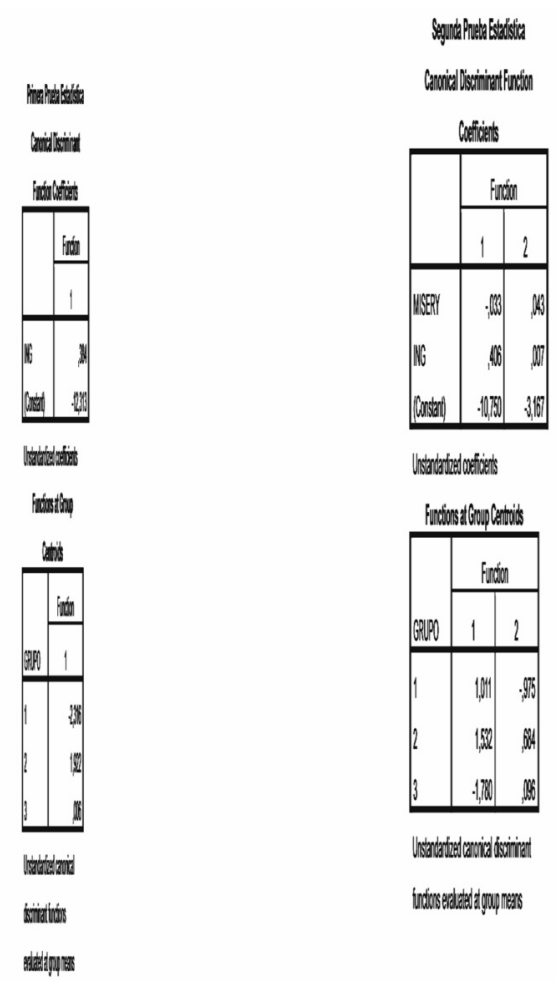




\section{Anexo F. Grupo pronosticado y probabilidades en otros grupos}

\begin{tabular}{|c|c|c|c|c|c|}
\hline Países & $\begin{array}{l}\text { Prueba } 1 . \\
\text { Grupo que } \\
\text { Pertenece }\end{array}$ & $\begin{array}{c}\text { Grupo } \\
\text { Pronostico }\end{array}$ & $\begin{array}{c}\text { Probabilidad de pertenecer } \\
\text { al Grupo } 1 \text { (ALBA) }\end{array}$ & $\begin{array}{c}\text { Probabilidad de pertenecer } \\
\text { al Grupo } 2 \text { (AP) }\end{array}$ & $\begin{array}{l}\text { Probabilidad de pertenecer } \\
\text { al Grupo } 3 \text { (Otros) }\end{array}$ \\
\hline Argentina & 3 & 2 & 0,00132 & 0,52742 & 0,47126 \\
\hline Bolivia & 1 & 1 & 0,66575 & 0,00165 & 0,3326 \\
\hline Brasil & 3 & 2 & 0,00024 & 0,73856 & 0,2612 \\
\hline Chile & 2 & 2 & 0 & 0,98472 & 0,01528 \\
\hline Colombia & 2 & 2 & 0,00082 & 0,59453 & 0,40465 \\
\hline Costa Rica & 2 & 2 & 0,00006 & 0,84663 & 0,15331 \\
\hline Cuba & 1 & 1 & 0,9853 & 0 & 0,01469 \\
\hline Ecuador & 1 & 1 & 0,71537 & 0,00116 & 0,28347 \\
\hline El Salvador & 3 & 3 & 0,37861 & 0,00801 & 0,61338 \\
\hline Guatemala & 3 & 3 & 0,11769 & 0,0377 & 0,84461 \\
\hline Honduras & 3 & 1 & 0,83336 & 0,00039 & 0,16626 \\
\hline México & 2 & 2 & 0,0004 & 0,68265 & 0,31694 \\
\hline Nicaragua & 1 & 1 & 0,939 & 0,00006 & 0,06094 \\
\hline Panamá & 2 & 2 & 0,00001 & 0,92014 & 0,07985 \\
\hline Paraguay & 3 & 3 & 0,05738 & 0,07208 & 0,87055 \\
\hline Perú & 2 & 3 & 0,00218 & 0,45372 & 0,54409 \\
\hline R. Dominicana & 3 & 3 & 0,03009 & 0,11804 & 0,85188 \\
\hline Uruguay & 3 & 3 & 0,00211 & 0,45922 & 0,53867 \\
\hline Venezuela & 1 & 1 & 0,92854 & 0,00008 & 0,07138 \\
\hline
\end{tabular}

\begin{tabular}{|c|c|c|c|c|c|}
\hline Países & $\begin{array}{l}\text { Prueba } 2 . \\
\text { Grupo que } \\
\text { Pertenece }\end{array}$ & $\begin{array}{c}\text { Grupo } \\
\text { Pronostico }\end{array}$ & $\begin{array}{l}\text { Probabilidad Grupo } 1 \\
\text { (MERCOSUR) }\end{array}$ & $\begin{array}{l}\text { Probabilidad Grupo } 2 \\
\text { (AP) }\end{array}$ & $\begin{array}{l}\text { Probabilidad Grupo } 3 \\
\text { (Otros) }\end{array}$ \\
\hline Argentina & 1 & 1 & 0,8205 & 0,17935 & 0,00015 \\
\hline Bolivia & 3 & 3 & 0,00138 & 0,00086 & 0,99776 \\
\hline Brasil & 1 & 2 & 0,46804 & 0,53127 & 0,00069 \\
\hline Chile & 2 & 2 & 0,0682 & 0,93175 & 0,00004 \\
\hline Colombia & 2 & 2 & 0,22861 & 0,74835 & 0,02304 \\
\hline Costa Rica & 2 & 2 & 0,23269 & 0,76619 & 0,00112 \\
\hline Cuba & 3 & 3 & 0,12967 & 0,00095 & 0,86937 \\
\hline Ecuador & 3 & 3 & 0,00054 & 0,00041 & 0,99905 \\
\hline El Salvador & 3 & 3 & 0,00272 & 0,00311 & 0,99417 \\
\hline Guatemala & 3 & 3 & 0,0528 & 0,05492 & 0,89228 \\
\hline Honduras & 3 & 3 & 0,00167 & 0,00049 & 0,99784 \\
\hline México & 2 & 2 & 0,13515 & 0,83607 & 0,02878 \\
\hline Nicaragua & 3 & 3 & 0,00027 & 0,00007 & 0,99966 \\
\hline Panamá & 2 & 2 & 0,08586 & 0,91261 & 0,00153 \\
\hline Paraguay & 1 & 3 & 0,20615 & 0,18884 & 0,60501 \\
\hline Perú & 2 & 2 & 0,25597 & 0,68679 & 0,05724 \\
\hline R. Dominicana & 3 & 3 & 0,31481 & 0,31934 & 0,36584 \\
\hline Uruguay & 1 & 2 & 0,35834 & 0,61719 & 0,02446 \\
\hline Venezuela & 1 & 1 & 0,96012 & 0,00318 & 0,0367 \\
\hline
\end{tabular}


JHONER PERDOMO. Estadístico, Candidato a Doctor en Ciencias Sociales por la Universidad Central de Venezuela (UCV). Profesor de Estadística en la Escuela de Sociología UCV (2014-2016). Miembro asociado de la American Statistical Association (2008-2015) y Royal Statistical Society (2016). Consultor Independiente en Estadística y en la Prevención del Lavado de Dinero.

LUIS DANIEL ÁlVAREZ VANEGAS. Doctor en Ciencias Sociales. Magister en Ciencia Política y en Educación. Especialista en Sistemas Electorales. Licenciado en Estudios Internacionales, en Comunicación Social y en Educación. Cursante del Postdoctorado en Ciencias Sociales. Cursante de la Especialización en Estudios Políticos y Constitucionales. Actualmente Director de la Escuela de Estudios Internacionales de la UCV. Integrante del Grupo Ávila y del Instituto Cultural Venezolano Israelí.

MAURICIO PHÉLAN C. Sociólogo de la Universidad Central de Venezuela (UCV) y Especialista en Análisis de Datos para las Ciencias Sociales de la misma casa de estudios. Doctor en Sociología por la Universidad de Barcelona, España. Se desempeña como Profesor Titular de la Escuela de Sociología de la Facultad de Ciencias Económicas y Sociales UCV.

SARY LEVY-CARCIENTE. Economist with expertise in the field of financial macroeconomics. PhD in Development Studies, Master in International Economics and Professional Specialization in Administrative Sciences. Board member of the National Academy of Economics Sciences. Dean of the Economics and Social Science Faculty at Universidad Central de Venezuela (2008-2011). www.sarylevy.com

Recibido:01/04/2016

Aceptado: 30/11/2016 